\title{
Primary seminoma arising in the middle mediastinum: A case report
}

\author{
JING XU $^{1 *}$, JINGLAN ZHAO $^{2 *}$, SHAOQING GENG ${ }^{1}$, QI WANG ${ }^{1}$, PENGFEI WANG $^{3}$, \\ CHUNLING ZHANG ${ }^{3}$, XIONGZENG ZHU ${ }^{4}$ and YOUXIN JI ${ }^{3}$ \\ Departments of ${ }^{1}$ Pathology, ${ }^{2}$ Surgery and ${ }^{3}$ Internal Medicine, Qingdao Central Hospital, \\ The Second Affiliated Hospital of Qingdao University Medical College, Qingdao, Shandong 266042; \\ ${ }^{4}$ Department of Pathology, Fudan University Shanghai Cancer Center, Shanghai 200032, P.R. China
}

Received March 31, 2015; Accepted April 5, 2016

DOI: $10.3892 / 01.2016 .4575$

\begin{abstract}
Primary mediastinal seminoma often occurs in the anterior mediastinum of young males. It is unusual for the tumor to originate in the middle or posterior mediastinum, and such cases have rarely been reported in the English literature. The present study reports the case of a 52-year-old man with a $3.0-\mathrm{cm}$ primary seminoma arising in the middle mediastinum. The patient presented with the symptoms of cough and chest tightness. Fluorine-18 fluorodeoxyglucose-positron emission tomography $\left({ }^{18} \mathrm{~F}-\mathrm{FDG}\right.$-PET) scans revealed unique abnormal FDG uptake in the middle mediastinum. A mediastinoscopy was performed and integral excision was found to be difficult. A biopsy was performed and the histological examination revealed a primary seminoma. Following 4 cycles of a standard bleomycin, etoposide and cisplatin chemotherapy regimen, and chest irradiation at a total dose of 40 Gy in 20 fractions, the tumor exhibited a partial response, decreasing in size, and FDG uptake was no longer observed on ${ }^{18} \mathrm{~F}$-FDG-PET scan. The last follow-up date was April 2016 and the patient has remained disease-free for 20 months.
\end{abstract}

\section{Introduction}

Primary mediastinal germ cell tumors (GCTs) are relatively rare, and are often complicated by various pathological types and characteristics. Seminoma is one kind of such tumors. Primary mediastinal seminoma usually occurs in young males, and the tumor often locates to the anterior mediastinum. Primary mediastinal germ cell tumors (GCTs) are

Correspondence to: Dr Youxin $\mathrm{Ji}$, Department of Internal Medicine, Qingdao Central Hospital, The Second Affiliated Hospital of Qingdao University Medical College, 127 Siliunan Road, Qingdao, Shandong 266042, P.R. China

E-mail: kevinji78@163.com

*Contributed equally

Key words: mediastinum, seminoma relatively rare, accounting for only approximately $1 \%-3 \%$ of all GCTs (1) and seminoma is the second most common mediastinal GCTs. Approximately $90 \%$ of primary malignant mediastinal GCTs occur in male subjects in the 2nd, 3rd, 4th decades of life (2).The histogenesis of primary seminoma of the anterior mediastinum is unknown; it has suggested that it develops from extragonadal, potentially biphasic germ cells left within the embryonic thymus. In terms of survival, the prognosis of seminoma is usually well, 5-year survival rate $\sim 75 \%$. Nonseminoma has a worse prognosis compared with seminoma. Cases with pleural dissemination or metastasis also have a worse prognosis. It would be possible to improve the prognosis with the establishment of a standard treatment regimen, development of new agents for the treatment of tumors resistant to current chemotherapy regimens, and detection of more tumors in the early stage (3).

Here we present an extremely rare case of primary seminoma arising in the middle mediastinum of a 52-year-old man.

\section{Case presentation}

A 52-year-old male was admitted to Qingdao Central Hospital (Qingdao, China) in June 2014, due to a cough and chest tightness. Thoracic computed tomography (CT) scans showed a $3.0 \times 2.3-\mathrm{cm}$ mass located in the space between the trachea and the superior vena cava (Fig. 1A). Fluorine-18 fluorodeoxyglucose-positron emission tomography $\left({ }^{18} \mathrm{~F}-\mathrm{FDG}-\mathrm{PET}\right)$ scans revealed unique abnormal FDG uptake in the mediastinal tumor, with a clear margin and uniform inner density without calcification (Fig. 1B-F). Serum $\alpha$-fetoprotein and $\beta$-human chorionic gonadotropin levels were tested and found to be within the normal ranges. The patient's leukocyte level was a little bit higher than the normal range $\left(9.8 \times 10^{9}\right.$; normal range $\left.3.5-9.5 \times 10^{9}\right)$. All other blood tests were in normal ranges. The patient had suffered from type II diabetes mellitus (DM) for 10 years and also had a family history of DM.

A mediastinoscopy was performed and the tumor was found located on the right side of trachea, behind the vena cava and beneath the azygos vein (Fig. 2). Tumor tissues were in close proximity and adhered to the superior vena cava, and integral excision was difficult. A biopsy was implemented and histological examination revealed a primary seminoma, with 
A
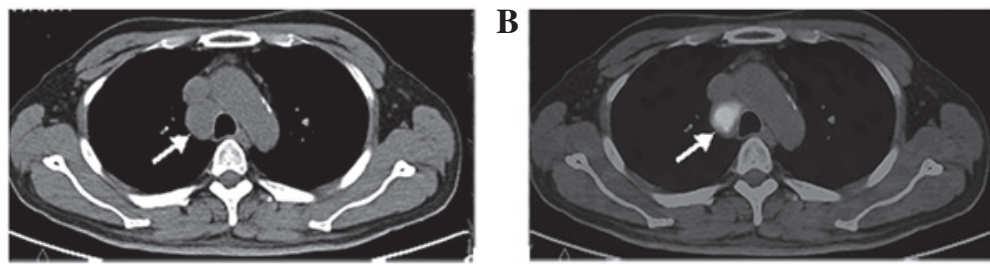

C
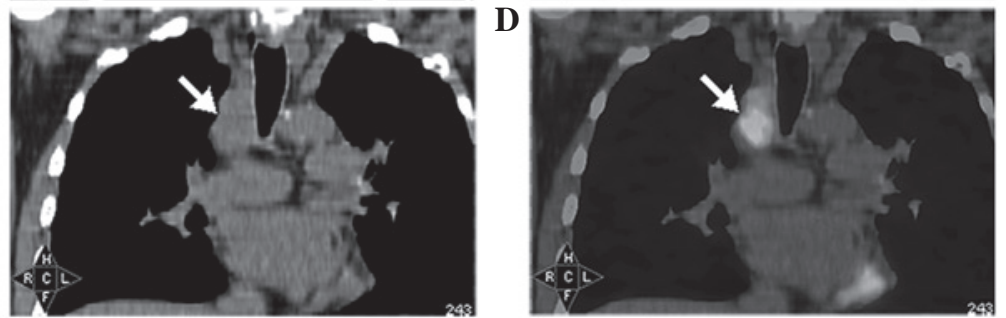

$\mathbf{E}$

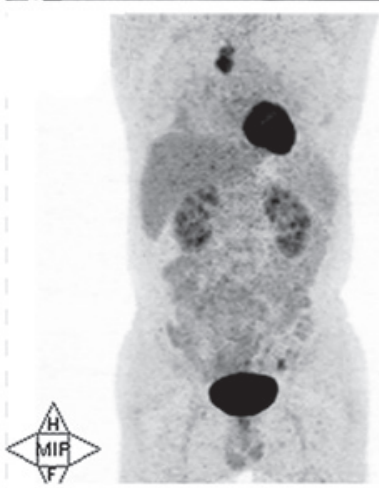

$\mathbf{F}$

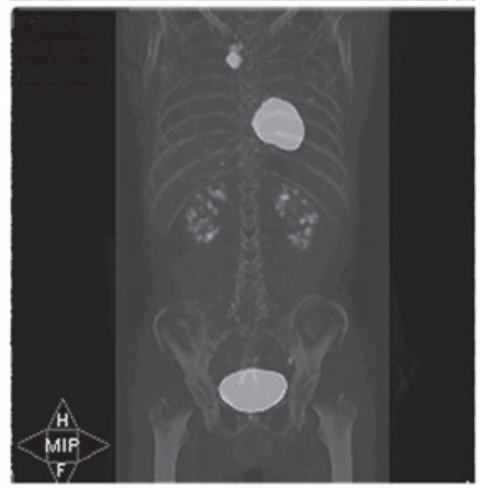

Figure 1. (A) Thoracic computed tomography scans demonstrating a mass located in the space between the trachea and superior vena cava. (B) ${ }^{18} \mathrm{~F}$ FDG-PET scans revealed the unique abnormal FDG uptake mediastinal tumor; (C and D) 18F FDG-PET/CT scans revealed the mass located before the trachea and behind vena cava. The tumor was oval in shape and had a clear margin. (E and F) There was no other site with increased FDG uptake.

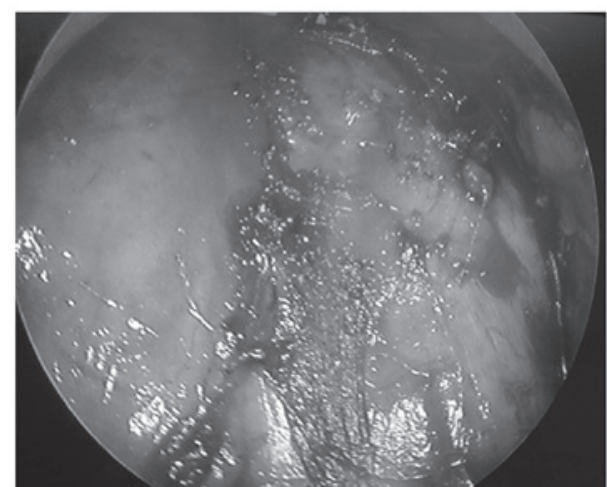

Figure 2. Mediastinoscopy view showing a well-demarcated tumor located on the right side of the trachea, behind the vena cava and beneath the azygos vein.

some sheets of round and polygonal tumor cells surrounded by lymphocytes on the paraffin-embedded sections (Fig. 3A). The tumor cells had a clear or granular cytoplasm, coarse-clumped chromatin and prominent nucleoli with frequent mitoses. The diagnosis of seminoma was established by the immunohistochemical studies of the neoplastic tissue. The tumor was positive for placental alkaline phosphatase (PLAP) (Fig. 3B), which is used as a representative surface marker of seminoma. As there were no tumors found at the thymus or testicles, or at any other site upon ${ }^{18} \mathrm{~F}$-FDG-PET, a primary middle mediastinal seminoma was diagnosed.

At 2 weeks post-biopsy, chemotherapy was started with the bleomycin, etoposide and cisplatin regimen $[25 \mathrm{mg}$ intravenous
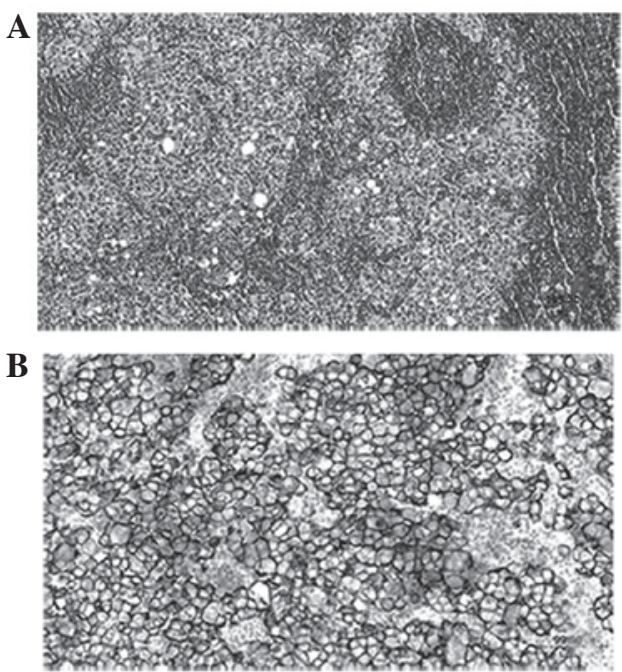

Figure 3. (A) Microscopically, sheets of round and polygonal tumor cells were found to be surrounded by lymphocytes (hematoxylin and eosin stain; original magnification, x100). (B) Immunohistochemical studies showing cytoplasmic staining with placental alkaline phosphatase (original magnification, $\mathrm{x} 200$ ).

(i.v.) bleomycin once a week for 3 weeks, $100 \mathrm{mg}$ i.v. etoposide on days 1-4 and $60 \mathrm{mg}$ i.v. Naida platinum on days 1-2], for 4 cycles, followed by conformal intensity-modulated radiation therapy (2 Gy/day; total, $40 \mathrm{~Gy}$ ). A partial response was achieved, as the tumor decreased in size and no FDG uptake was apparent on ${ }^{18} \mathrm{~F}$-FDG-PET after finishing the 
chemoradiotherapy. The patient has not received any further treatment after finishing 4 cycles of chemotherapy and 40 Gy radiotherapy, regular follow-up was initiated thereafter. The last follow-up date was April 2016 and the patient has remained disease-free for 20 months.

\section{Discussion}

Extragonadal germ cell tumors (GCTs) are typically located along the midline of the trunk, and seminoma is the second most common type of all mediastinal GCTs. Mediastinal seminoma is frequently located in the anterior mediastinum involving the thymus. Upon reviewing the English literature in PubMed (http://www.ncbi.nlm.nih.gov/pubmed), only 3 cases of seminoma that primarily originated from the middle mediastinum were found (4-6). As certain anterior mediastinal seminomas may enlarge and extend into the middle mediastinal compartment, it is a challenge to distinguish the initial site for large tumors. In the present study, ${ }^{18}$ F-FDG-PET scans assisted in locating the unique mass in the middle mediastinum, and also played an important role in clinical staging and the evaluation of therapy effects.

These extragonadal tumors are believed to develop from germ cell precursors that become arrested during embryological migration and survive in ectopic locations. Approximately $90 \%$ of primary malignant mediastinal GCTs occur in male subjects in the third decade of life. In the three studies of seminoma that occurred in middle mediastinum (4-6), all cases were in men and the age ranged from 25 to 69 years old, with a mean age of 53 years old when including the present patient.

Primary mediastinal seminoma typically occurs with non-specific symptoms, including chest pain or pressure, dyspnea, hoarseness, dysphagia and fever. Rarely, the tumor may cause superior vena cava syndrome (7) and severe back pain (8). With regard to the three aforementioned studies, all cases were either asymptomatic or presented with dull retrosternal chest pain (4-6). The present patient complained of a cough and chest tightness, which was due to the closeness of the tumor and/or its compression of the trachea. The symptoms were closely associated with the location and size of the tumor.

The differential diagnosis of mediastinal seminoma includes a range of metastatic and primary malignant mediastinal neoplasms. The possibility of metastasis from a testicular primary tumor should always be considered. In the present case, ${ }^{18} \mathrm{~F}$-FDG-PET played an important role in distinguishing the primary tumor from a metastasis. Considering the unusual location of the tumor and the age of the patient, poorly-differentiated carcinoma, embryonal carcinoma, thymic carcinoma, lymphoma and mesothelioma were also differential diagnostic considerations (9). The morphological overlap between embryonal carcinoma or seminoma and a poorly-differentiated carcinoma (e.g., pulmonary, thymic or metastatic) is significant. Cytokeratin and cluster of differentiation (CD)30 are characteristically coexpressed in embryonal carcinoma, but are not specific to only this tumor. Other primary carcinoma markers, including thyroid transcription factor-1 (TTF-1; lung) and CD5 (thymic), may also be useful in this setting (9). The present case was negative for CD30, TTF-1 and CD5, but positive for PLAP, so the possibility of embryonal carcinoma, metastatic lung cancer or thymic carcinoma was excluded. Primary mediastinal (thymic) large B cell lymphoma (PMBL), lymphoblastic lymphoma (LBL), anaplastic large cell lymphoma (ALCL) and classic Hodgkin's lymphoma (CHL) may potentially mimic embryonal carcinoma or seminoma (9). The markers of lymphoma are complicated, with the main distinguishing markers including CD20 and CD23 (PMBL), CD34 and terminal deoxynucleotidyl transferase (LBL), epithelial membrane antigen and anaplastic lymphoma kinase (ALCL); and CD15 and paired box protein Pax-5 (CHL). As all the aforementioned markers were negative, the possibility of a number of lymphomas was also excluded. Although PLAP has traditionally been the preferential marker for the objective verification of germ cell origin (mostly seminoma) in the setting of an undifferentiated neoplasm, it has been found that for extragonadal seminoma, octamer-binding transcription factor $3 / 4$, activator protein $2 \gamma, \mathrm{D} 2-40$ and c-kit are equivalently superior to PLAP (10).

Patients with early-stage mediastinal seminoma are curable by complete surgical resection followed by radiation therapy (4,000-4,500 cGy). All other advanced-stage patients should receive initial cisplatin-based chemotherapy. In those individuals who are considered not to be good candidates for combination chemotherapy and who have tumors in the mediastinum, radiation therapy is an acceptable initial treatment (11).

In conclusion, primary seminoma in the middle mediastinum is extremely rare, but may be cured by combined modalities. Oncologists should be aware of this disease and continue to shed further light on its early diagnosis. Completely surgical resection with adjuvant chemo-irradiation was the cornerstone for the successful treatment of this case.

\section{References}

1. Nichols CR. Mediastinal germ cell tumors: clinical feature and biologic correlates. Chest 99: 472-479, 1991.

2. Knapp RH, Hurt RD, Payne WS, et al: Malignant germ cell tumors of the mediastinum. J Thorac Cardiovasc Surg 89: 82-89, 1985.

3. Yano M and Fujii Y. Results of surgical treatment for pimary germcell tumors of the mediastinum. Nihon Geka Gakkai Zasshi 107: 278-83, 2006 [In Japanese].

4. Nakamura H, Hashimoto T, Kusama H, Sudoh A, Adachi H, Yagyu H, Kishi K, Oh-ishi S and Matsuoka T: Primary seminoma in the middle mediastinum: Intern Med 43: 1191-1193, 2004.

5. Kiffer JD and Sandeman TF: Primary malignant mediastinal germ cell tumor: A study of 11 cases and a review of the literature. Int J Radiat Oncol Biol Phys 17: 835-841, 1989.

6. Kitami A, Suzuki K, Suzuki S and Hori G: Primary seminoma in the middle mediastinum: Case report in a 69-year-old male. Jpn J Clin Oncol 28: 142-144, 1998.

7. Xu X, Sun C, et al: A case of mediastinal seminoma presenting as superior vena cava syndrome. Intern Med 51: 1269-1272, 2012.

8. Kaako A, et al: Mediastinal extragonadal seminoma presenting as severe back pain in a young male. Tenn Med 104: 41-45, 2011.

9. McKenney JK, Heerema-McKenney A and Rouse RV: Extragonadal germ cell tumors: A review with emphasis on pathologic features, clinical prognostic variables, and differential diagnostic considerations. Adv Anat Pathol 14: 69-92, 2007.

10. Iczkowski KA, Butler SL, Shanks JH, Hossain D, Schall A, Meiers I, Zhou M, Torkko KC, Kim SJ and MacLennan GT: Trials of new germ cell immunohistochemical stains in 93 extragonadal and metastatic germ cell tumors. Hum Pathol 39: 275-281, 2008.

11. Hainsworth JD and Greco FA: Germ cell neoplasms and other malignancies of the mediastinum. Cancer Treat Res 105: 303-325, 2001. 